\title{
The Compact Muon Solenoid Experiment

\section{Beam tests of the trigger and digital processing electronics for the electromagnetic calorimeter of the CMS experiment}

\author{
R. Benetta, M. Hansen, B. Lofstedt, J. Varela \\ CERN, Geneva, Switzerland \\ P. Lecomte, F. Nessi-Tedaldi \\ ETH, Zürich, Switzerland
}

A. Almeida, E. Machado, R. Nóbrega, J.C. da Silva, S. Silva

LIP, Lisbon, Portugal

M. Bercher, J. Bourotte, L. Buiron, Ph. Busson, L. Faurlinia), D. Lecouturier, P. Matricon

LPNHE IN2P3-CNRS, Palaiseau, France

\begin{abstract}
A prototype of the trigger and digital processing electronics for the electromagnetic calorimeter of the CMS experiment, coupled to a prototype of the $\mathrm{PbWO}_{4}$ crystal calorimeter, was tested during summer 96 in the H4 beamline at the CERN SPS. A very successful operation was achieved for this system, which runs in synchronous and pipelined mode at the LHC clock frequency, and performs the basic trigger and data acquisition functions needed in the CMS electromagnetic calorimeter. The performance of the trigger front-end electronics is well within the established requirements: a highly efficient bunch crossing identification ( $>99.9 \%)$, a good trigger energy resolution $(\sigma / E \sim 9 \% / \sqrt{E} \oplus$ $2 \%)$ and a highly efficient electron cluster shape identification $(\sim 99 \%)$ have been achieved. The FERMI digitising system based on a dynamic analog compressor and a sampling ADC showed a very good performance, in particular the energy resolution for $150 \mathrm{GeV}$ electrons was $0.54 \%$, equal to the resolution obtained with a conventional charge integration ADC system.
\end{abstract}

\footnotetext{
a) Now at CENBG IN2P3-CNRS
} 


\section{Introduction}

In this paper we report on the results of a prototype test of the trigger and digital processing electronics for the electromagnetic calorimeter of the CMS experiment [1]. The data were collected during summer 96 in the $\mathrm{H} 4$ beamline at the CERN SPS, where a prototype of the $\mathrm{PbWO}_{4}$ crystal calorimeter of CMS was under test.

The readout electronics of the CMS electromagnetic calorimeter is required to digitise the analog signals with a precision of 12 bits and to cover a dynamic range above $2^{16}$. The trigger electronics is required to compute the trigger primitives (trigger tower energy sums, and fine-grain cluster shape variables) at a rate of $40 \mathrm{MHz}$ and to provide the correct bunch crossing assignment, performing the first stage of the CMS synchronous and pipelined calorimeter triggering [2].

In order to match the required dynamic range to the high performance 12-bit, $40 \mathrm{MHz}$ sampling ADCs available today, the analog signal is preprocessed by a dynamic non-linear compressor or by a multi-gain linear circuit with range determination. In either cases, the digital information is linearised and calibrated after the ADC using a memory look-up table, from where it follows parallel data acquisition and trigger paths. For data acquisition purposes, data from each channel are stored in a pipeline memory with a length matching the first level trigger latency. In the trigger path, data from the crystals forming a trigger tower are processed to extract the trigger tower primitives at the correct clock period. The trigger primitive data (together with the corresponding data from the hadronic calorimeter) are then transferred through high speed serial links to the regional trigger processors, which analyse sliding windows of $3 \times 3$ trigger towers in the search for isolated electron and photon candidates, and perform the energy sums needed for the jet and missing transverse energy triggers [4].

The main goals of the test reported here were to validate the concepts used in the digital signal processing on data from the high precision CMS crystal calorimeter, both for triggering and data acquisition purposes. A front-end trigger and readout system with the basic functionality needed to process the CMS electromagnetic calorimeter data was put in operation at the LHC clock frequency in a test beam environment. A matrix of $6 \times 6 \mathrm{PbWO}_{4}$ crystals, corresponding to one barrel trigger tower, ${ }^{1)}$ was equipped with prototypes of the FERMI readout system [5], consisting of a dynamic non-linear analog compressor, a 10-bit sampling ADC, a lineariser look-up table and a readout pipeline memory. In the trigger chain, individual channel samples were summed in strips of six channels and then filtered in order to extract the energy and timing information from the sampled pulses, using circuits provided by the FERMI collaboration [6]. The strip energy data were processed by a prototype of the trigger primitive generator, which computes the trigger tower energy sum and a variable characterising the transverse profile of the electromagnetic shower [7]. The trigger data were stored in pipeline memories for readout by the data acquisition system. The system operated in synchronous and pipelined mode at $40 \mathrm{MHz}$ clock frequency, and data were collected using electron beams with energies ranging from 15 to $150 \mathrm{GeV}$.

\section{Experimental Setup}

The crystals used in the calorimeter prototype matrix were truncated pyramids with a length of $230 \mathrm{~mm}(25.8$ radiation lengths) and a $20.5 \times 20.5 \mathrm{~mm}^{2}$ front face, pointing to a focus located at a distance of $1430 \mathrm{~mm}$ on the beam line. They closely approximated the crystals to be used near $\eta=0$ in the baseline CMS design. The crystal axes were tilted by $3^{\circ}$ to the beam direction in both transverse coordinates to correspond to the intended configuration of the CMS electromagnetic calorimeter. The crystal matrix was water cooled and the temperature was stabilised at $18^{\circ} \mathrm{C}$. The temperatures of the front and rear surfaces of the crystals were continuously monitored and written to tape with the other data. The results presented in this paper are not corrected for temperature variations, considered to be negligible within the duration of the data taking.

Optical fibres feed monitoring light pulses to the front of the crystals allowing for tracking of calibration changes. During the data taking periods changes in the calibration were negligible. Two different APDs from EG\&G and Hamamatsu were used for light detection. Most of the data collected in this test were obtained with the beam incident on a crystal equipped with an EG\&G APD. The signals from the APDs went into fast, low-noise preamplifiers with a peaking time of $35 \mathrm{~ns}$, and then were transmitted through $80 \mathrm{~m}$ cables from the beam area to the control room. A more detailed description of the detector set-up can be found in reference [8].

The analog signals were split by an active circuit in order to feed in parallel the electronic system under test and a standard charge integration 13-bit ADC system. Measurements showed that the splitters did not add any significant

\footnotetext{
1) The geometry of the electromagnetic calorimeter described in this paper corresponds to the detector version presented in the CMS Technical Proposal [1]. Recently, a new geometry was defined [3], in which one trigger tower is composed of $5 \times 5$ crystals of larger dimensions, such that the $\Delta \eta \times \Delta \phi$ trigger tower coverage remains unchanged.
} 
noise to the signal. At splitter output the pulses are $175 \mathrm{~ns}$ wide (FWHM) and span the range 0-2 V. The ADC gate width was set at $300 \mathrm{~ns}$.

The FERMI prototype readout electronics was implemented in 6U VME modules, each processing three channels. Each FERMI channel is composed of a compressor ASIC, a custom-designed 10 bit sampling ADC and a digital channel ASIC performing the linearisation and memory functions.

The compressor performs a non-linear transformation, compressing the signal according to an approximately piecewise linear transfer function, producing on the output a signal in the $0-2 \mathrm{~V}$ range. This circuit forms a sum of the outputs from four linear amplifiers with gains of approximately 18.5, 1.3, 0.14 and 0.09 and upper cutoffs on the input voltage of 60, 470, 1200 and $2000 \mathrm{mV}$ respectively. Figure 1 shows the measured compression curve of one of the circuits. The compression functions of different circuits are similar but not identical, so that the functions have to be computed individually for all channels.

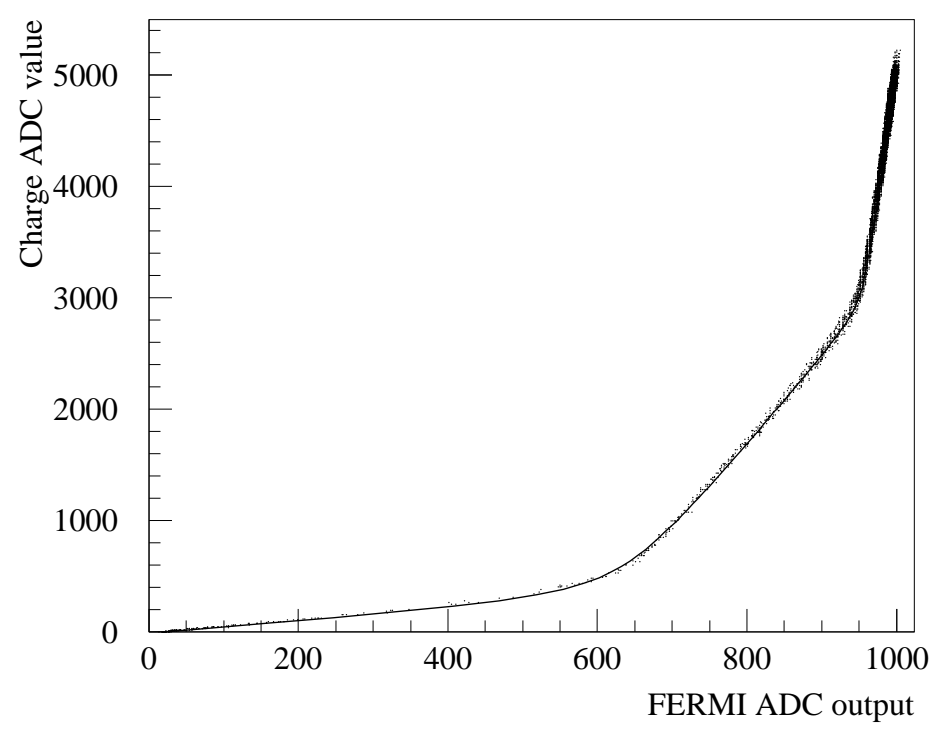

Figure 1: Compression function measured for the central channel in the crystal matrix. A fitted analytical function is superimposed on the data points

Despite the fact that the 10-bit ADC does not meet the CMS physics requirements, it was appropriate for this test since the beam energy was limited to $150 \mathrm{GeV}$. Decompression at the ADC output is performed by a programmable look-up table with 16 bit output. After linearisation and pedestal subtraction, the energy equivalent of the least significant bit is $30 \mathrm{MeV}$ and saturation is reached at $200 \mathrm{GeV}$, for samples taken at pulse maximum. The dynamic range of the system is about $2^{13}$.

The circuit adds 6 bit Hamming error correction code to each 16 bit data sample, and stores the 22 bit frame in a pipeline memory at clock frequency. For the purposes of this test, the circuit was programmed so that the pipeline input is stopped at beam trigger arrival and its content is made available for VME readout. The pipeline was programmed to contain 32 signal samples and the trigger timing was adjusted in such a way that the sample 17 is at pulse maximum. About 10 samples before the pulse are available for pedestal determination in an event by event basis (see figure 8).

The three channels in a FERMI board are added sample by sample using a dedicated adder ASIC and the result is available at clock frequency at the board front panel. This three channel sum is the first step in the computation of the CMS calorimeter trigger primitives.

The outputs of two FERMI boards, corresponding to a strip of six crystals, are fed into the filter module. After summation of the two inputs, the time profile of the strip energy pulse is filtered in the Filter ASIC. The circuit performs the convolution $F_{i}^{\prime}=\sum_{j=0}^{5} \phi_{j} \cdot F_{i+j}$, where $F_{i+j}$ represents a window of six consecutive samples of the input pulse and $\phi_{j}$ represents a vector of six programmable coefficients. In parallel, a peak finder selects the maximum sample of $F^{\prime}$ and inhibits the output of the other samples. The latency of the filter circuit is of eight clock periods, including six clocks for data input. In figure 2 we show three different events collected with the 
filter programmed as described in the figure's caption.
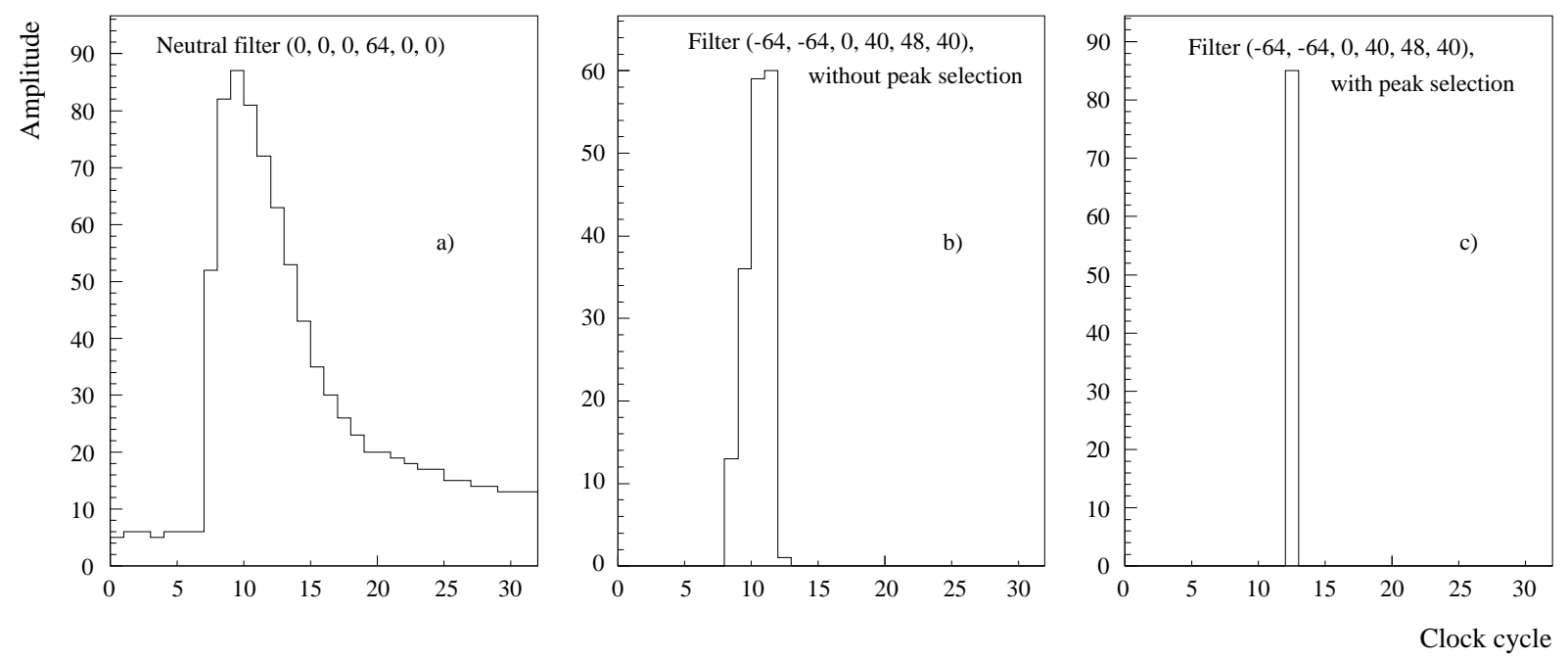

Figure 2: Three different events collected with the following filter programming: a) neutral filter, all filter coefficients identical to zero except one; b) filter performing pedestal subtraction and energy estimation; c) the same as $\mathrm{b}$ with peak selection

The trigger primitive generator system (TPG) receives input from six filter modules, which correspond to the energy values in the six crystal strips. The inputs are coded to 10-bit integer numbers with a LSB value of $250 \mathrm{MeV}$ in order to cover a $255 \mathrm{GeV}$ dynamic range.

The TPG is a set of three different VME 6U boards and a special board to connect them [9]: 1) the system board (SB); 2) the processor board (PB); and 3) the optical link board. In this test, only the first two boards have been used. The PB has been designed to emulate the whole functionality of the Trigger Primitive ASIC which is needed for the fine grain calorimeter trigger algorithm in CMS [7]. The processing unit makes use of three L-Neuro 2.3 chips from Philips [10]. The SB controls the PB, stores in the readout FIFO's the input data as well as the results of the computations done by the PB and handles the VME interface [11]. The readout FIFO's behave as a pipeline memory of programmable length (a length of 32 was used in the test). Self-test and boundary scan facilities were also implemented in the SB for test purposes.

The PB makes the summation $S$ of the six input signals and performs the fine grain algorithm as follows:

- it computes all five sums of two consecutive input signals $x_{i}\left(x_{0}+x_{1}, x_{1}+x_{2}, \ldots, x_{4}+x_{5}\right)$;

- it finds $S_{2}$, the maximum value of the five sums, and gives its location;

- it compares the variable $R=S_{2} / S$ to a threshold value and sets the electron/photon bit accordingly.

The PB operates at $40 \mathrm{MHz}$ and has a latency of 17 clock periods. A common clock and the beam trigger signal are distributed to all modules in the system. In the present setup, the phase of the beam trigger is not synchronised with the clock, so that the time jitter is uniformly distributed within a clock period. The beam trigger timing is adjusted with programmable delays in steps of $25 \mathrm{~ns}$ so that the content of all pipeline memories are synchronised. In the first data taking period, data in the FERMI pipelines and in the TPG FIFO's were found to be desynchronised by one clock period in $20 \%$ of the events. The source of the problem was later identified and corrected for. The beam trigger was built with a coincidence of beam scintillator counters with a cross-section of $2 \times 2 \mathrm{~cm}^{2}$. At each beam trigger, data in the pipeline memories were read, together with the data from the charge integration ADC's as well as from beam chambers. About 15000 events were taken for each beam energy point $(15,35,50,100,150 \mathrm{GeV})$. At the start of each data taking period, calibration data were collected with the $50 \mathrm{GeV}$ beam incident in each one of the 36 used crystals.

\section{Performance of the trigger system}

The trigger data generated by the filter module and by the TPG show that the expected performance has been achieved. In the following we present the results obtained in the operation of the trigger system. 
After calibration runs, a first estimate of the decompression functions, ADC pedestals and calibration constants was made, so that look-up tables with values appropriate for the TPG on-line operation could be loaded. A simplified version of the methods described in the next section was used for this purpose, which nevertheless guaranteed a level of precision of a few percent, sufficient for the trigger measurements. Under these conditions, the trigger tests were performed in the situation typical at experiment setting-up, when the calibration and linearisation constants are not yet well known.

Very high efficiency in the bunch cross identification is one of the main concerns for a proper operation of the trigger systems in the LHC experiments. In the tested system, this functionality is provided by the Filter ASIC which converts the pulse samples spanning six clock periods into a single sample different from zero, with a fixed phase relative to the beam crossing.

At LHC, the ADC clock is expected to be synchronous with the beam interaction with a precision better than $1 \mathrm{~ns}$. This situation does not occur in the test beam, since the beam particle timing is random relative to the clock. In these conditions, the events were classified into different time jitter windows, and the time distribution of the filter output as a function of the jitter was studied. The time jitter was estimated from the derivative of the pulse shape at the maximum sample, a variable called $\alpha$. The variable $\alpha$, computed for the signal collected in the hit crystal, has a distribution almost flat in the interval -0.20 to 0.26 as shown in figure 3 , so that in first approximation an interval in $\alpha$ of 0.01 corresponds to a jitter of $0.55 \mathrm{~ns}$.

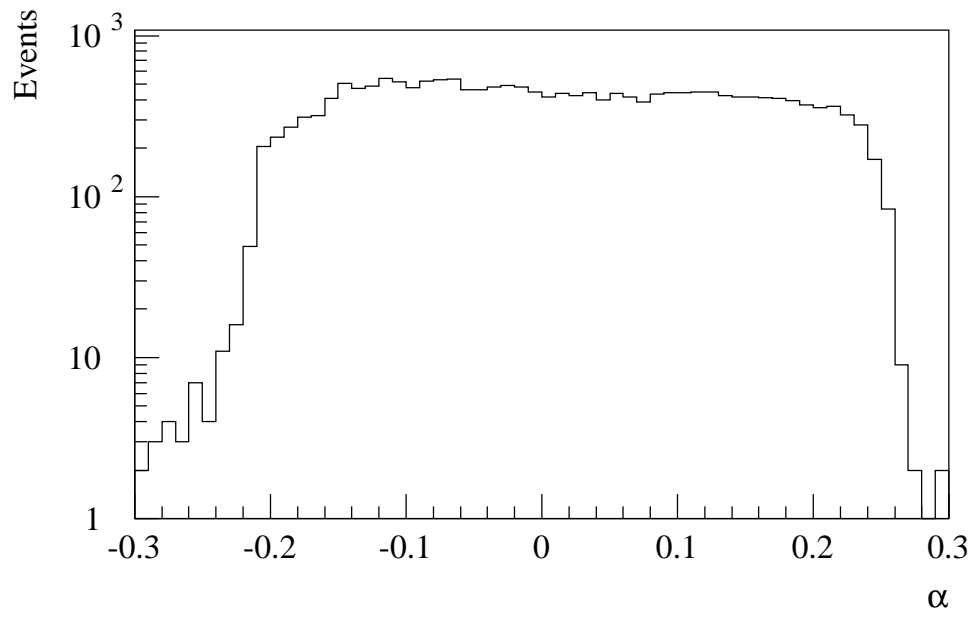

Figure 3: Distribution of $\alpha$ for the pulses measured in the hit crystal and for the events collected with electrons of $50 \mathrm{GeV}$

The results are presented in figure 4, which shows the distribution of the pipeline memory address where the filter output was recorded for different intervals of $\alpha$. The events selected in the $\alpha$ interval between -0.02 and 0.02 , which corresponds to a time jitter window of about $2 \mathrm{~ns}$, have the filter output always at the same address. This means that, within the limited statistics available, a constant phase between the beam trigger signal and the filter output is maintained. When the jitter window becomes larger we start to observe a spread in the filter output timing.

The resolution of the trigger energy measurement is an important parameter since it determines the sharpness of the efficiency curves at trigger threshold. Figure 5 shows the resolution of the energy computed by the TPG system. For comparison, the trigger energy resolution obtained off-line with optimised decompression tables, calibration constants and filter coefficients is presented in the same plot, showing an improvement of a factor two compared to the online results.

The Monte Carlo simulation results have shown that the efficiency of the fine grain algorithm is rather sensitive to the value of the threshold in the cluster shape variable $R$ [12], making the measurement of the $R$ distribution for real electrons an important target for this test. The results are shown in figure 6 . The measurement of $R$ performed with beam data agrees reasonably with the Monte Carlo expectations. In any case the distribution sits well above the threshold value assumed in the algorithm simulations $\left(R_{\mathrm{th}}=0.89\right)$ [12]. For this cut, the electron identification efficiency estimated with the test beam data is of the order of $99 \%$. 

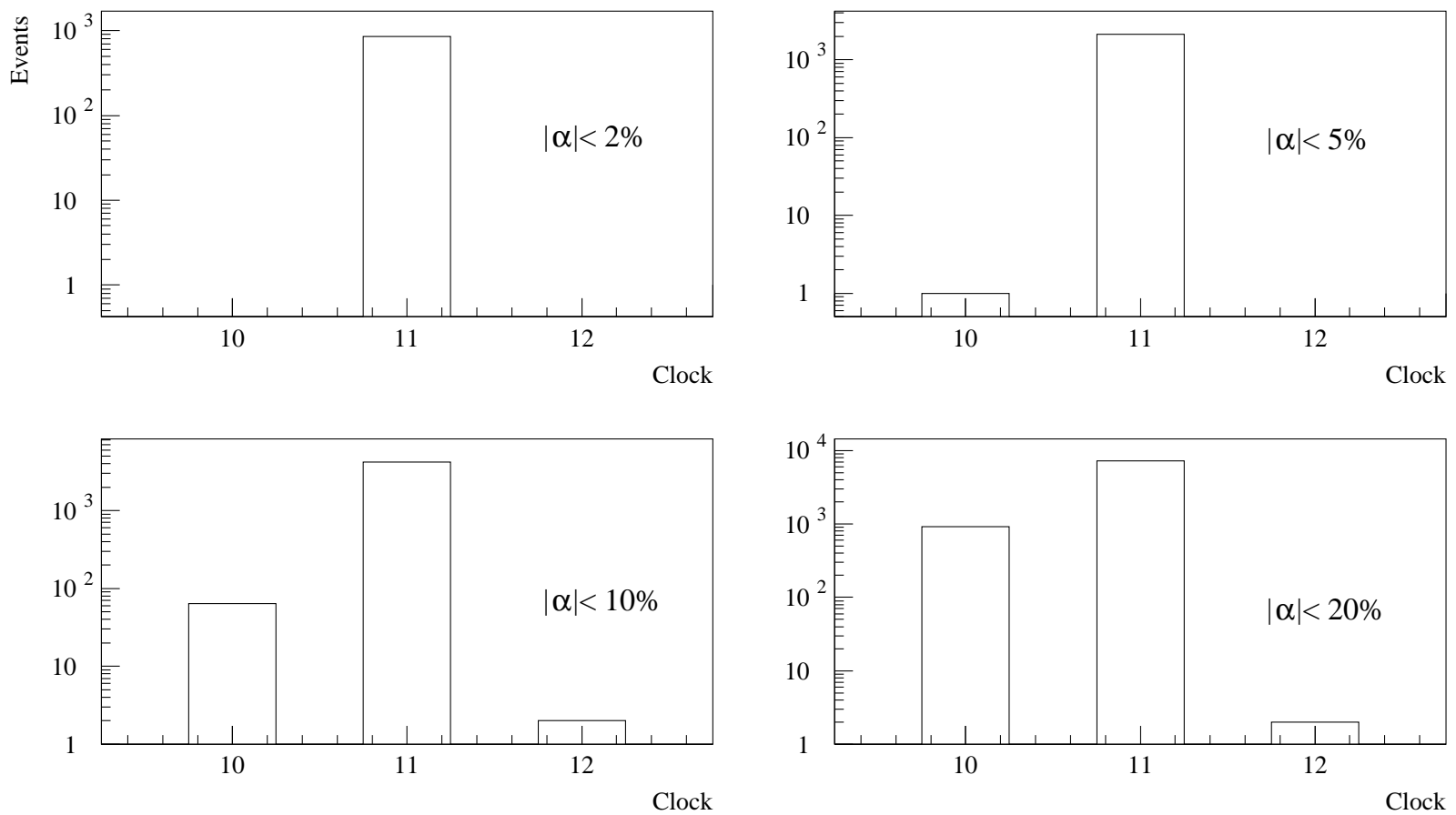

Figure 4: Time distribution of the filter output for different intervals of $\alpha$. The events selected in the $\alpha$ interval between -0.02 and 0.02 (about one thousand) peak in the same clock period, showing that for a time jitter of the order of $2 \mathrm{~ns}$, the efficiency of bunch crossing identification is larger than $99.9 \%$

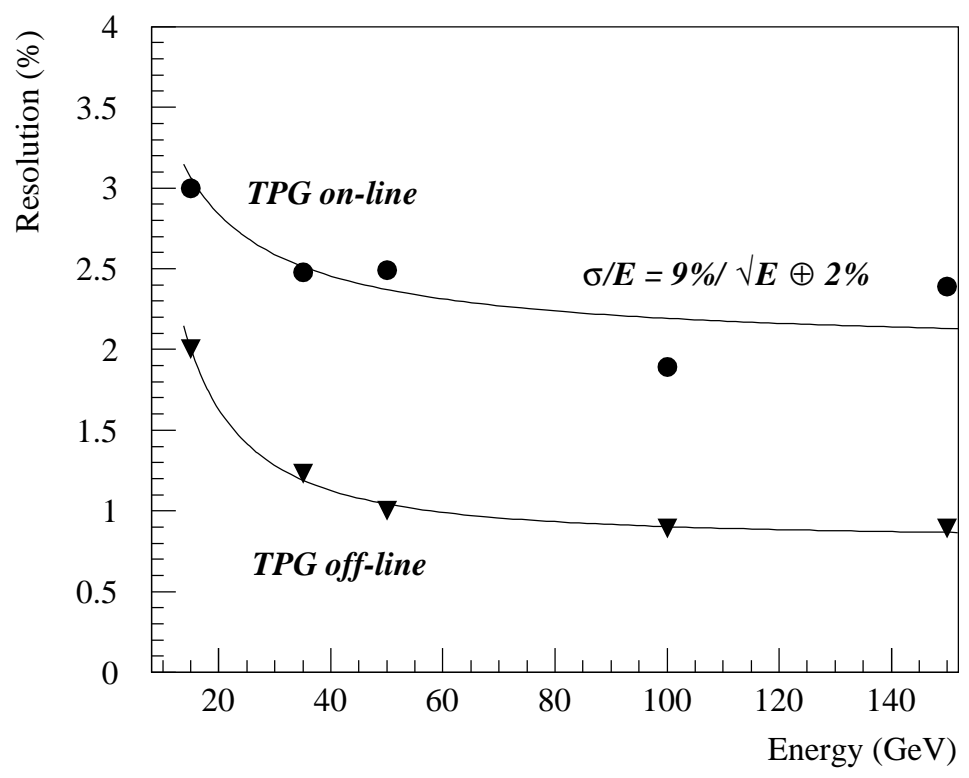

Figure 5: Trigger energy resolution. The on-line results are well represented by the function $9 \% / \sqrt{E} \oplus 2 \%$, shown in the plot 


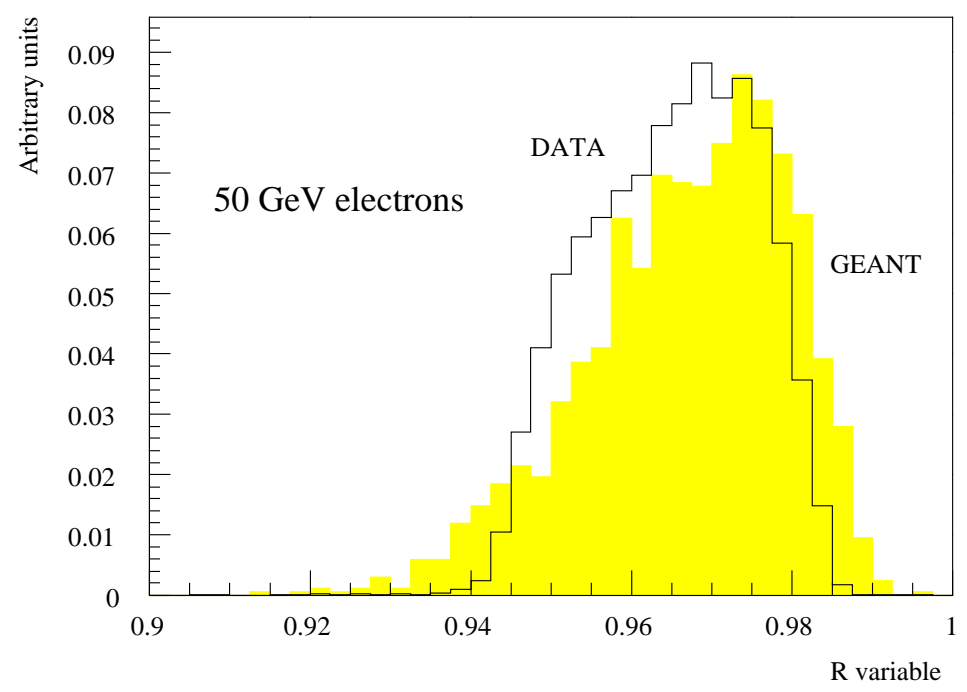

Figure 6: Distribution of the cluster shape variable $R$

\section{Off-line energy measurements}

The data collected with the FERMI system in the beam test have been analysed off-line in order to get the best possible measurement of the electrons energy. The main issues in the analysis were the linearisation of the digitised compressor data and the optimisation of an energy estimator out of the pulse samples.

The data produced in parallel by the charge integration ADC's have been used with a two-fold purpose. Firstly, the charge integration ADC data were analysed independently in order to provide reference energy measurements. Secondly, the data were used to compute the decompression tables using them as an estimator of the signal amplitude at the compressors input.

For this analysis, only the data collected in the central $3 \times 3$ crystals were used.

In the CMS experiment, the computation of the decompression functions would be a non-trivial task. Real electrons could eventually be exploited for this purpose. However, the lack of statistics at the higher end of the spectrum would prevent a correct coverage of the complete dynamic range. A special system capable of injecting precise charges at the compressor inputs could be used to compute the decompression functions. The performance of the whole method would nevertheless depend critically on the stability of the components involved.

The problem of energy estimation based on the FERMI data can be stated in the following way: what is the procedure which gives a single value $E$, the energy of the showering particle, given the set of $9 \times 32$ compressed values $f_{i}^{k}$ (for $k=1$ to 9 and $i=1$ to 32 ) recorded for each individual event? One can think of a general procedure from which one can obtain directly $E$ as a complex function of the $f_{i}^{k}$ (maybe a neural network inspired method for example). In this paper, a more conventional approach is adopted, in order to solve the energy estimation problem.

In a first step, all sample values are "linearised" in such a way that the compressed sample values are replaced by quantities proportional to the corresponding charge ADC value which is also recorded for each channel of the matrix. Then, combining $n$ (to be determined) "linearised" samples $\left(\mathcal{F}_{i}^{k}\right)$ inside a window (to be chosen) around the maximum of the time frame, a single number $\left(q_{F}^{k}\right)$, which characterises the energy released in each crystal, is obtained. Specifically, weighted sums were used to extract the nine $q_{F}^{k}$ values, according to the following formula:

$$
q_{F}^{k}=\sum_{i=l}^{l+n-1} w_{i}^{k} \mathcal{F}_{i}^{k}
$$

Using such a procedure, two elementary sub-problems remain:

1. how to compute the $\mathcal{F}_{i}^{k}$ from the $f_{i}^{k}$ ?

2. what are the best choices for $n, l$ and the $w_{i}^{k}$ ?

Moreover, the $q_{F}^{k}$ are constructed in such a way that they are expressed in the same units as the corresponding 
charge ADC values. Using the same calibration coefficients $c_{k}$ as the ones determined for the charge ADC, the energy is then computed in a final step, using the simple summation $E=\sum_{k=1}^{9} c_{k} q_{F}^{k}$.

\subsection{Analysis of charge ADC}

In order to extract the calibration constants, the drift chamber information that provided us with an estimate of the electron impact position $(x, y)$ in the matrix was used. The crystal "centre" is defined as the region which gives the maximum response. All events located in a $2 \times 2 \mathrm{~mm}^{2}$ window around this position were used to compute the calibration constants and as well as the energy resolution. A set of about 15 thousand events per crystal was used to extract these constants.

Beam-off events were used in order to compute charge ADC noise. Table 1 shows the noise value for each channel of the matrix. The computed dispersion for the sum of nine crystals was found to be equal to $119 \mathrm{MeV}$. This value is about $40 \%$ larger than the quadratic sum which amounts to $85 \mathrm{MeV}$. This effect has been tracked to the presence of correlation between the different electronics channels. In the rest of this analysis we will retain the value of $119 \mathrm{MeV}$ as the noise introduced by electronics.

\begin{tabular}{|c|c|c|c|c|c|c|c|c|c|}
\hline crystal number & 1 & 2 & 3 & 4 & 5 & 6 & 7 & 8 & 9 \\
\hline noise in $\mathrm{MeV}$ & 31 & 20 & 25 & 32 & 36 & 24 & 23 & 21 & 38 \\
\hline
\end{tabular}

Table 1: Individual channel noise

With this set of calibration constants, the energy resolution for energy runs $E=35,50,100$ and $150 \mathrm{GeV}$, displayed in table 2, was extracted. This table shows that for $E_{\mathrm{BEAM}}=150 \mathrm{GeV}$ the relative energy resolution reaches a value of $0.54 \%$.

\begin{tabular}{|c|c|c|c|c|}
\hline $\mathrm{E}_{\text {BEAM }}(\mathrm{GeV})$ & 35.00 & 50.00 & 100.00 & 150.00 \\
\hline $\mathrm{E}_{\text {SUM }}(\mathrm{GeV})$ & 34.99 & 50.13 & 99.93 & 149.92 \\
\hline$\sigma_{\text {SUM }}(\mathrm{MeV})$ & 349 & 412 & 602 & 812 \\
\hline $\begin{array}{c}\sigma_{\text {SUM }}(\mathrm{MeV}) \\
\text { noise subtracted }\end{array}$ & 328 & 394 & 590 & 803 \\
\hline
\end{tabular}

Table 2: Energy resolution obtained with the charge ADCs

\subsection{Analysis of FERMI ADC}

\subsubsection{Baseline estimation and subtraction}

Thirty two compressed samples were recorded in such a way that the maximum sample is located at the sampling clock tick number 17. This allows to perform an analysis of the baseline which can suffer from variations on an event by event basis. The baseline was computed as the mean value of 3 samples located at the beginning of the event time frame, where the influence of the signal itself is not present.

\subsubsection{Solution to sub-problem 1}

Before dealing with the digital processing (sub problem 2) we start examining the problem linked to the presence of a compression stage in the electronics chain. Experimental constraints as well as dispersions of the different electronic channels have to be taken into account at this point. At SPS the arrival time of electrons during a burst is random in regard to the $40 \mathrm{MHz}$ sampling clock of the FERMI system. The relative phase $\Delta t$ between the maximum of the signal and the nearest clock tick was not known at the acquisition level. This fact will have to be taken into account when reconstructing the energy of one event with a phase different from 0 . The strategy adopted was to correct for this effect by "offline" shifting the recorded frame, after linearisation of the samples, in order to have a zero phase. As earlier said, the true value $\Delta t$ was not known at the time the event was recorded, so we were obliged to develop a procedure to extract the $\Delta t$ value from the event itself. This would have been an easy task if the knowledge of the pulse shapes of the different crystals would have been perfect, as well as the knowledge of the analog compression functions. Distortion introduced by cables, as well as different conditions of measurement 
of the compression functions in laboratory prevent us from doing this. The good correlation between the charge ADC and the FERMI measurements was the key point to resolve all these problems at the same time.

The whole problem of linearisation of samples in presence of jitter effect as well as experimental constraints previously presented is equivalent to find $9 \times 32$ functions (called hereafter "decompression tables") which allow us to pass from the compressed sample values $f_{i}^{k}(\Delta t \neq 0)$ to $\mathcal{F}_{i}^{k}(\Delta t=0)$ with a priori unknown $\Delta t$.

To first order, the decompression tables are considered independent of $\Delta t$ and of the sample considered. "First order" decompression tables $T_{1}^{k}$ were built using the correlation of the charge ADC measurement with the compressed sample with the maximum value of the pulse. This choice was mainly driven by the fact that the jitter affects the maximum sample value by a bias proportional to the second derivative of the pulse shape around its maximum. An example of such a first order table was given in figure 1, for the central crystal of the matrix.

Transformation of the time frame by means of the $T_{1}^{k}$ tables gives access to a first order linearised time frame. Using a fitting procedure, the first order linearised time frame on a subset of events with energy in the range $[2.5 \mathrm{GeV}, 3.5 \mathrm{GeV}]$ is adjusted to the function

$$
\xi\left(\alpha, \beta, t_{\max }, t\right)=\left\{\begin{array}{ll}
A\left[\frac{\beta}{\alpha}\left(t-t_{\max }+\frac{\alpha}{\beta}\right)\right]^{\alpha} e^{-\beta\left(t-t_{\max }\right)} & \text { if } t \geq t_{\max }-\frac{\alpha}{\beta} \\
0 & \text { if } t<t_{\max }-\frac{\alpha}{\beta}
\end{array},\right.
$$

where $A$, the ratio $\alpha / \beta$ and $t_{\max }$ represent respectively the amplitude, the rising time of the signal and the time where the signal is maximum.

During this fitting procedure, $A, t_{\max }, \alpha$ and $\beta$ vary freely. As a consequence of dispersion of the various signal shapes, values for the ratio $\alpha / \beta$ in the range $[67 \mathrm{~ns}, 89 \mathrm{~ns}]$ were obtained. Comparison of the $t_{\max }$ value with the position of the maximum sample gives an estimate $(\widetilde{\Delta t})$ of the relative phase $\Delta t$. The mean pulse shapes of events with $\widetilde{\Delta t}$ in the range $[-1 \mathrm{~ns}, 1 \mathrm{~ns}]$ were taken as the approximate pulse shapes before the analog compression. For these events, the consistency between the value of $t_{\max }$ and the rising time of the signal was checked. It was found that the values for $t_{\max }$ and $\alpha / \beta$ are in very good agreement as it should be for events with zero relative phase. In the rest of the analysis, the value of $\alpha / \beta$ was fixed in the fitting procedure, letting only $A$ and $t_{\text {max }}$ vary freely. Further comparison of these reference pulse shapes with the mean pulse shapes obtained in different bins of $\widetilde{\Delta t}$ different from zero allows to compute the "second order" tables $T_{2, i-i_{\max }}^{k}$, which will correct the first order time frame for jitter effects. This correction depends as previously said on the $\Delta t$ value and the relative position of the sample compared to the maximum sample inside the time frame. An example of the deviations from 1 of the second order table $T_{2}^{k=5}$ for the maximum sample is shown in figure 7 .

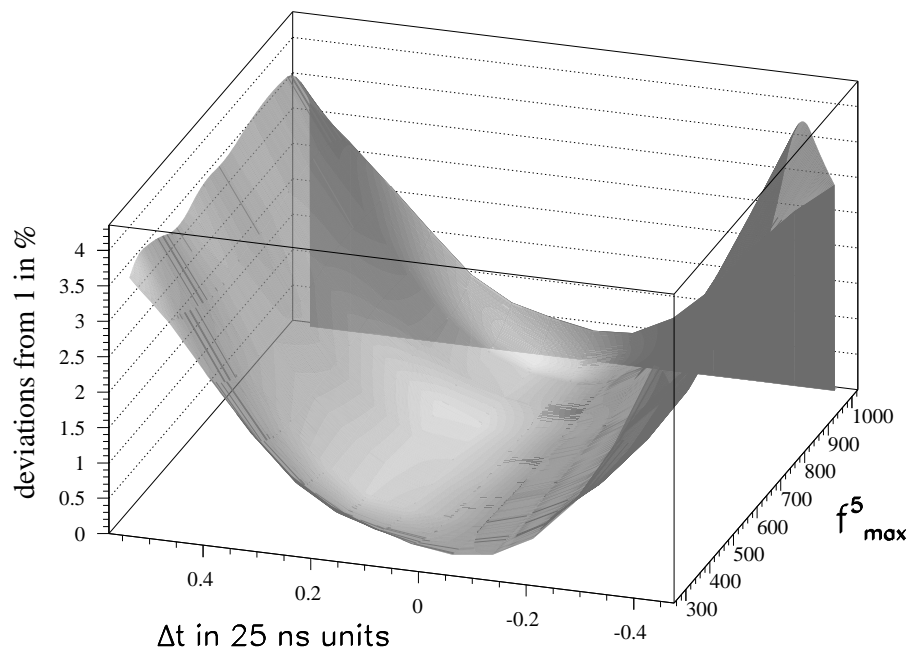

Figure 7: Deviations from unity of table $T_{2, i-i_{\max }}^{k}$ of the central crystal for $i=i_{\max }$ vs the relative phase and the compressed value 
In summary, the following three-step procedure has been followed to obtain the linearised time frame corrected for jitter effect:

- linearise the compressed samples neglecting sample number and relative phase $f_{i}^{k} \longrightarrow T_{1}^{k}\left(f_{i}^{k}\right)$

- extract from the first order linearised time frame the relative phase $\widetilde{\Delta t}$

- the time frame corrected for jitter effect is obtained by $f_{i}^{k} \longrightarrow \mathcal{F}_{i}^{k}=T_{1}^{k}\left(f_{i}^{k}\right) \times T_{2, i-i_{\max }}^{k}\left(f_{i}^{k}, \widetilde{\Delta t}\right)$

The differences between the first order time frame $T_{1}^{k}\left(f_{i}^{k}\right)$ and the time frame linearised and corrected for jitter effect, $\mathcal{F}_{i}^{k}$, can be seen in figure 8 for an event with a relative phase equal to $4.8 \mathrm{~ns}$.

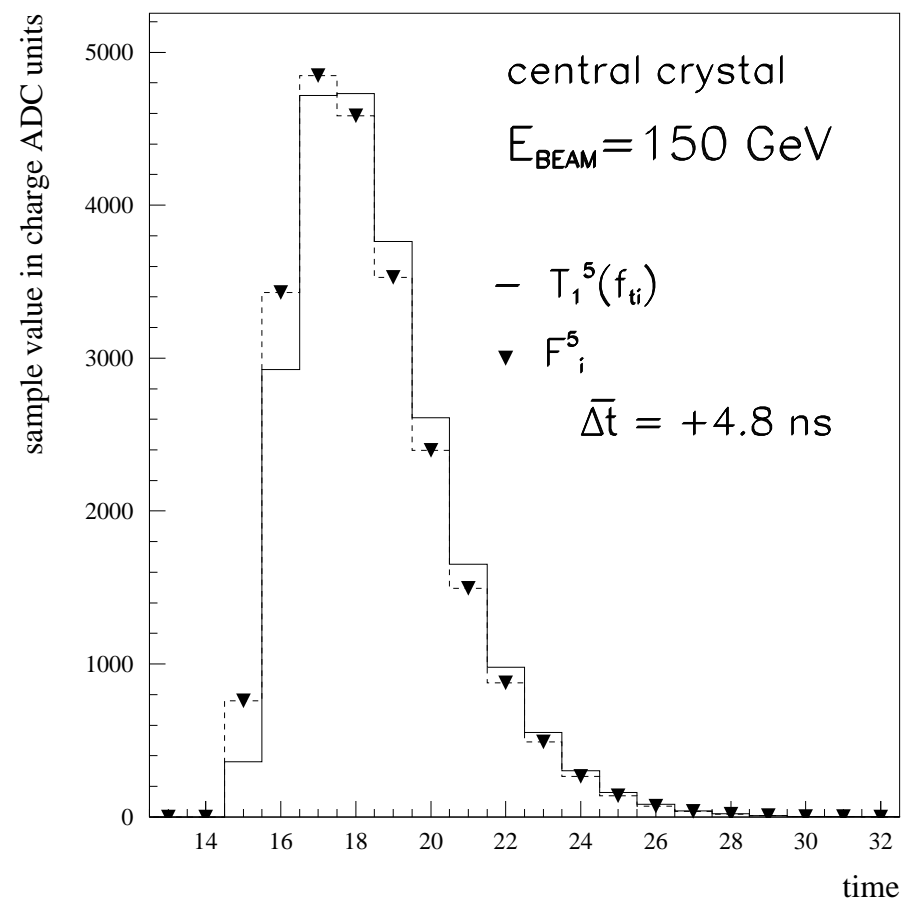

Figure 8: Effect of the jitter correction: the histogram represents the time frame after linearisation, the triangles represent the same time frame after correction of the jitter effect. The relative phase extracted from the time frame was $4.8 \mathrm{~ns}$

The consistency of the procedure has been checked in several ways. The fit to the individual shapes obtained after the first order decompression was used to extract the signal timing information $\widetilde{\Delta t}$. Relative to the hit channel (crystal with $k=5$ ) the timing of the eight neighbouring signals was found to have dispersions between 2.2 and $4.0 \mathrm{~ns}$. The larger dispersions were observed in the neighbouring channels with the smaller signal amplitudes corresponding to the shower tails. The degradation of the time determination for those channels is not significant when compared to the bunch separation at LHC. Therefore, no ambiguities in the beam crossing assignment of the signals forming an electromagnetic shower are to be expected. The linearity of the decompressed and corrected for jitter samples is very good for the nine channels. Over the full available energy range and for sample position inside the time frame between 15 and 20 which are the most relevant for extraction of energy, it was found that the maximum deviation from linearity is less than $0.3 \%$ for hit channel and less than $0.4 \%$ for channel 9 (worst channel).

\subsubsection{Solution to sub problem 2}

Sub problem 2 is, as defined above, related to the problem of digital processing of a sampled signal. Among the numerous possibilities to compute the equivalent charge ADC $q_{F}^{k}$ from the FERMI measurements the simplest one is to use the single value $\mathcal{F}_{i}^{k}$ for $i=i_{\max }$. This is obviously not the optimal use of information contained in the different samples. In particular, the resolution of this energy estimator is worse than the energy resolution obtained from the charge ADC's. Another possibility would be to build a "digital charge integrator", summing samples 
around the maximum with equal weights. Adding more samples is equivalent to add information, but this means also adding more noise.

The usual way to solve the problem of combining several measurements in presence of correlation, is to compute optimal weights taking into account the correlation matrix between the samples, in order to obtain the minimum value for the energy resolution. It was found that optimal weights depend on the crystal number, because of the different pulse shapes, as well as on the slightly different auto-correlation functions. It was also found that the weight values are depending on energy, but with a negligible effect on the resulting resolution. The total noise has been computed using the optimal weight values applied to event time frames recorded in absence of signal. Figure 9 shows the evolution of the computed noise for different values of the number of samples.

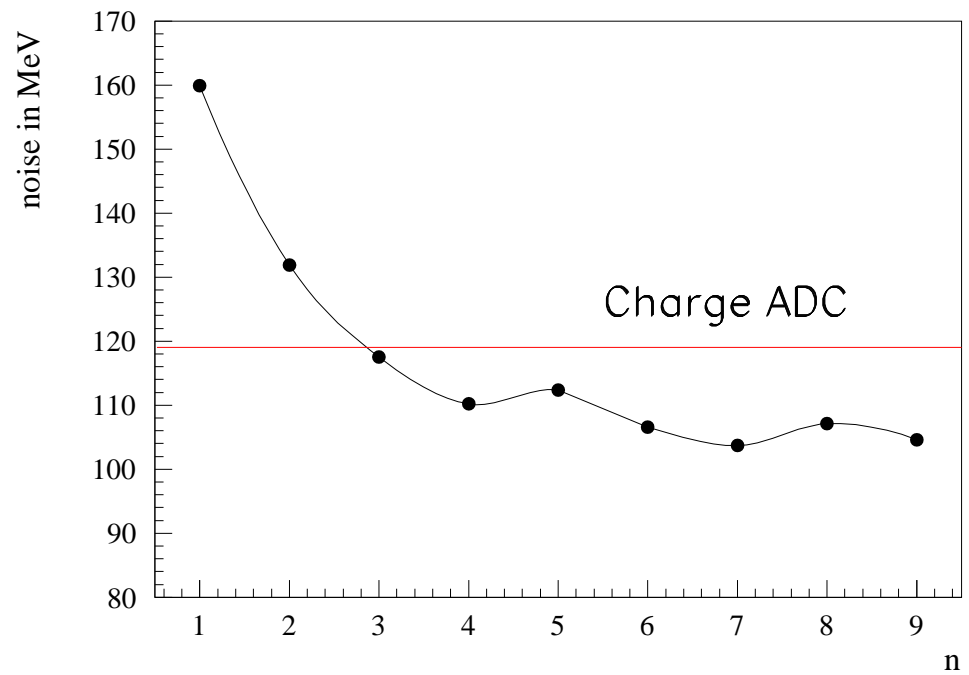

Figure 9: Evolution of the noise vs the number of samples used in the optimal weighted sum

As for the charge ADC total energy estimate, the energy of the matrix has been computed using the sum of the nine energy values of the crystals. This energy sum is represented by $E_{n}$, as it depends on the number $(n)$ of samples used in the optimal weighted sum. Evolution of the resolution of this estimator shows that an optimum is obtained for $n=4$, which corresponds to perform the weighted sum of samples from $i=i_{\max }-1$ to $i=i_{\max }+2$. The noise contributed by these samples amounts to $110 \mathrm{MeV}$, and was quadratically subtracted from the values obtained for the energy. The relative resolution can be parametrised with a stochastic term equal to $4.9 \%$ and a constant term equal to $0.34 \%$, which are very close to the equivalent values obtained with the charge ADC (5.1\% and $0.35 \%$ respectively). The two resulting curves are shown superimposed on figure 10 .

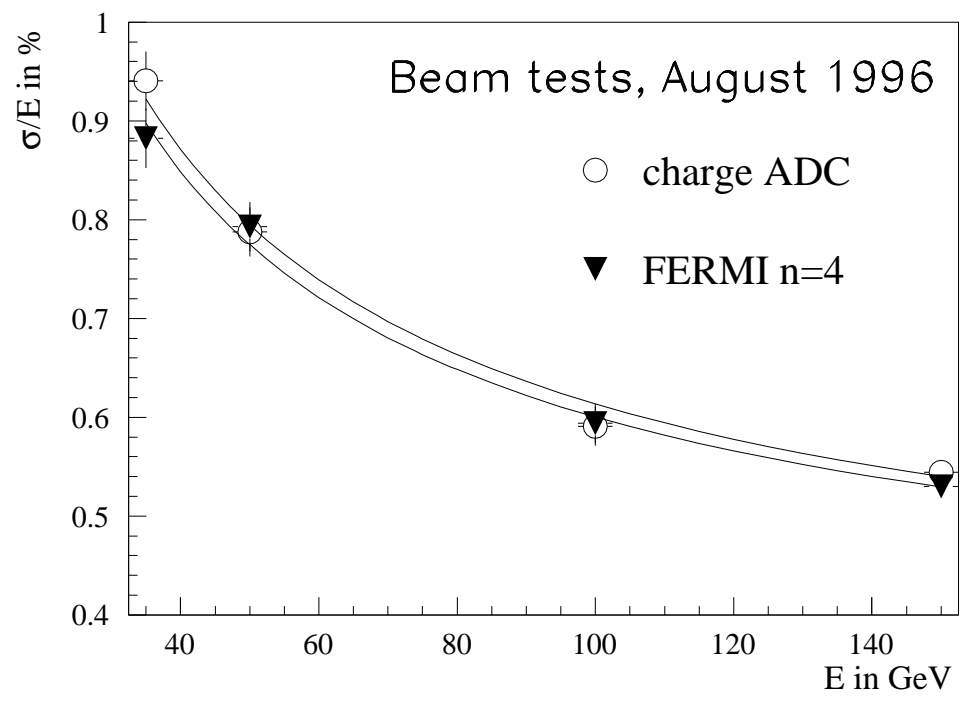

Figure 10: Energy resolution for the charge ADC system (circles) and for the optimal estimation from the FERMI system (triangles) 
The linearity of this energy estimator was found to be very good (the maximum deviation is lower than $0.22 \%$ on the whole energy range) and it can be concluded that this estimator perfectly fulfills the CMS requirements in terms of resolution and linearity of energy measurement of the ECAL clusters.

\section{Conclusions}

The test of prototypes of the trigger and digital processing electronics for the electromagnetic calorimeter of the CMS experiment described in this paper allows to draw the following conclusions:

- No major problems were found in the operation of the system, which runs in synchronous and pipeline mode at the LHC clock frequency, and performs the basic trigger and data acquisition functions needed in the CMS electromagnetic calorimeter;

- The performance of the trigger front-end electronics is well within the established requirements, in particular we have shown that highly efficient bunch crossing identification $(>99.9 \%)$, good trigger energy resolution $(\sigma / E \sim 9 \% / \sqrt{E} \oplus 2 \%)$ and highly efficient electron cluster shape identification $(\sim 99 \%)$ was achieved;

- The FERMI digitising system based on a dynamic analog compressor and a sampling ADC showed a very good performance, in particular the energy resolution for $150 \mathrm{GeV}$ electrons was $0.54 \%$, equal to the resolution obtained with the conventional linear and charge integration ADC system. However, we have concerns about the feasibility of a high precision measurement, where it is mandatory to keep track of eventual changes of the non-linear decompression curves, in a calorimeter with a number of channels of the order of one hundred thousand.

\section{References}

[1] CMS Technical Proposal, CERN/LHCC 94-38

[2] G. Heath, The CMS calorimeter trigger, Third Workshop on Electronics for LHC Experiments

[3] CMS Electromagnetic Calorimeter Project, Technical Design Report, CERN/LHCC 97-33

[4] J. Lackey et al., CMS Calorimeter Level 1 Trigger Conceptual Design, CMS/TN 94-284

[5] H.Alexanian et al., Nucl. Instr. Meth. A357 (1995) 306

[6] H.Alexanian et al., Nucl. Instr. Meth. A357 (1995) 318

[7] G.Heath et al., Preliminary specifications of the baseline trigger algorithms, CMS/TN 96-10

[8] E.Auffray et al., Beam tests of lead tungstate crystal matrices and a silicon strip preshower detector for the CMS electromagnetic calorimeter, to appear

[9] Ph.Busson et al., Trigger Primitives Boards, CMS/IN 96-8

[10] M.Duranton, L-Neuro 2.3: A VLSI for Image Processing by Neural Networks, Proceedings of MicroNeuro'96-Lausanne

[11] A.Almeida et al. TPB System Board Technical Documentation, CMS/IN 97-13

[12] R.Nóbrega, J.Varela, The CMS e/ $\gamma$ trigger; simulation study with CMSIM data, CMS/TN 96-21 